Check for updates

Cite this: Chem. Commun., 2017, 53,4108

Received 4th December 2016, Accepted 17th March 2017

DOI: $10.1039 / \mathrm{c} 6 \mathrm{cc} 09638 \mathrm{~g}$

rsc.li/chemcomm

\section{Self-assembly and redox induced phase transfer of gold nanoparticles at a water-propylene carbonate interface $\dagger$}

\author{
Evgeny Smirnov, (D) Pekka Peljo (D) and Hubert H. Girault (D) *
}

Citrate-stabilized gold nanoparticles were found to spontaneously self-assemble into a lustrous film at a bare water-propylene carbonate interface after vigorous shaking, due to the extremely low interfacial tension. The presence of an electron donor, tetrathiafulvalene, in the oil phase, led to the extraction of particles into the organic phase.

Interfaces between two immiscible liquids represent an excellent model system to study the self-assembly of various types of nanoparticles for many different applications. ${ }^{1-3}$ Liquid-liquid interfaces (LLIs) have a number of unique properties in comparison with solid-liquid interfaces: flexibility, self-healing properties, defect-free nature and, thus, a more homogeneous energetic profile. ${ }^{2,4}$

Over the years, many techniques and methods have been proposed to self-assemble nanoparticles (NPs) at liquid-liquid interfaces. Among them there are reducing the Coulombic repulsion between nanoparticles by addition of salts or "promoters" of aggregation, ${ }^{5,6}$ changing the charge of the nanoparticle core, ${ }^{7,8}$ as well as using solvent evaporation ${ }^{9}$ and covalent bonding between particles. ${ }^{10}$ Recently, the topic of nanoparticle self-assembly was extensively reviewed including at electrified interfaces. ${ }^{11,12}$

Previously, we have shown that citrate stabilized gold nanoparticles (citr@AuNPs) in aqueous solution easily interacted with tetrathiafulvalene (TTF) molecules dissolved in the adjacent oil phase. ${ }^{7}$ Interfacial Fermi level equilibration, in other words, oxidation of TTF to $\mathrm{TTF}^{+} \bullet$ and the concomitant accumulation of electrons on the AuNP ${ }^{13}$ were observed. In turn, $\mathrm{TTF}^{+}{ }^{\bullet}$ forms stacks of several $(n) \mathrm{TTF} / \mathrm{TTF}^{+} \bullet$ molecules with a delocalised charge through $\pi-\pi$ interactions, i.e. $\operatorname{TTF}_{n}^{z<n} \cdot{ }^{14,15}$ This makes TTF-capped AuNPs sticky and leads to the self-assembly of AuNPs into a lustrous nanofilm at various LLIs. We will call these nanoparticles TTF@AuNPs. Consequently, these nanofilms showed remarkable

Laboratoire d'Electrochimie Physique et Analytique,

Ecole Polytechnique Fédérale de Lausanne, Rue de l'Industrie 17,

CH-1951 Sion, Switzerland.E-mail: hubert.girault@epfl.ch

$\dagger$ Electronic supplementary information (ESI) available: Experimental details, interfacial tension measurements, estimation of AuNP charge, additional experiments and description of the model used for thermodynamic simulation. See DOI: $10.1039 / \mathrm{c} 6 \mathrm{cc} 09638 \mathrm{~g}$ self-healing of the metallic lustre after repeated shaking ${ }^{16}$ and certain mechanical properties (due to $\pi-\pi$ interactions between separated TTF@AuNPs). ${ }^{7}$

Here, we further investigate and extend the self-assembly of AuNPs to water-organic interfaces with low interfacial tension $\left(\gamma_{\mathrm{w} / \mathrm{o}}\right)$, such as a water-propylene carbonate (PC) interface. The present results rely on both experimental observations and thermodynamic modeling in accordance with the work of Flatte et al. ${ }^{17}$ which allows understanding the obtained results at a qualitative level.

As already reported, ${ }^{16}$ citr@AuNPs spontaneously self-assembled at a water-nitromethane $\left(\mathrm{MeNO}_{2}\right)$ interface without TTF molecules. $\gamma_{\mathrm{w} / \mathrm{MeNO}_{2}}$ was determined to be $16 \mathrm{mN} \mathrm{m}^{-1}$. Further exploration of various water-organic solvent systems leads us to the water-PC interface, which possesses an extremely low interfacial tension of $2.95 \mathrm{mN} \mathrm{m}^{-1}$ as determined by pendant drop measurements (see Section S2 in the ESI $\dagger$ ). Since PC has quite a large solubility in water (ca. $17.5 \mathrm{wt} \%^{18,19}$ to $c a .25 \mathrm{wt} \%^{20}$ ), saturated solutions of PC in water and water in PC were used for interfacial tension measurements.

A low value of the interfacial tension is a reason of the immediate self-assembly of $32 \mathrm{~nm}$ citr@AuNPs into a lustrous nanofilm upon vigorous shaking of an aqueous solution in contact with a pure PC phase (Fig. 1A, right). While shaking, the red color of the initial AuNP solution turned bluish grey (Fig. 1A, middle), which is a sign of aggregation at the interface. Similar color changes were observed previously, ${ }^{7,16}$ when DCE was used as the organic phase.

Fig. 1B shows UV-Vis spectra of the initial solutions and of the obtained nanofilm (see Section S1 in the ESI $\dagger$ ). The aqueous solution of citr@AuNPs had only one distinct peak at $522 \mathrm{~nm}$ (black bar), which corresponds to the surface plasmon resonance, SPR (Fig. 1B). Once AuNPs were assembled into a nanofilm at the water-PC interface in a $10 \mathrm{~mm}$ square quartz cell, the SPR-peak shifted from 522 (black bar) to $548 \mathrm{~nm}$ (green bar) and an intense surface plasmon coupling (SPC)-band appeared with its peakposition at $c a .717 \mathrm{~nm}$.

This corroborates the previously published results on optical properties of nanofilms at various liquid-liquid interfaces. ${ }^{16}$ 
(A)

(B)
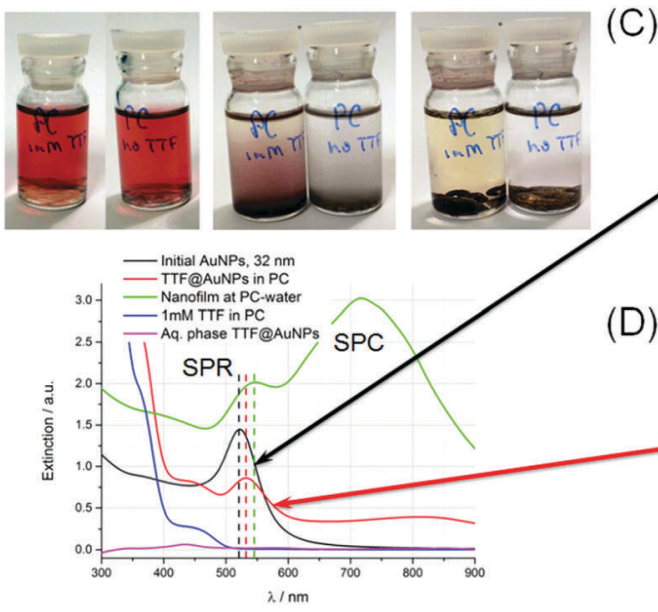

(D) sт

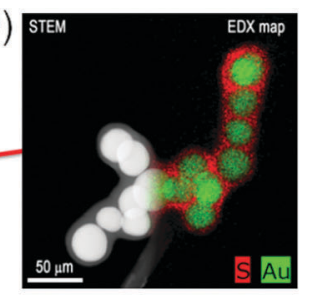

(E)

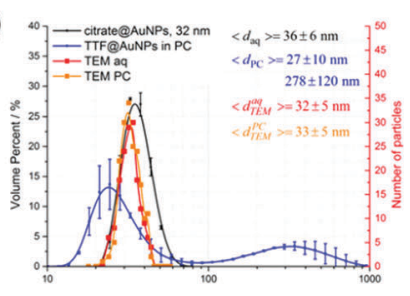

(F)

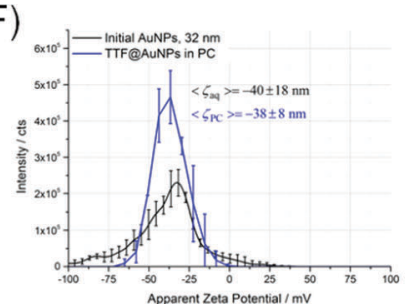

Fig. 1 Self-assembly at a LLI with low interfacial tension in the presence and absence of TTF (the lipophilic electron donor). (A) Photographs of vials with the aqueous phase on top and PC oil at the bottom. From left to right: before, right after shaking, and after complete phase separation with and without TTF in the PC phase. (B) UV-Vis spectra of the initial solutions, the spectra of the aqueous and PC phases after complete phase separation and the extinction spectra of the nanofilm formed at the water-PC interface. High resolution (S)TEM images of (C) as-prepared citr@AuNPs and (D) TTF@AuNPs with a chemical (EDX) map highlighting the presence of the TTF/TTF ${ }^{+}$layer (details are given in Section 3 of the ESI $\dot{\dagger}$ ). Comparison of the initial aqueous citr@AuNPs solution with TTF@AuNPs solution in PC: (E) DLS data showing aggregates formation in PC and (F) $\zeta$-potential measurements.

There are two main contributions to the observed large red-shift of the SPR-peak: (i) the change in the dielectric constant of the surrounding medium and (ii) depolarization factors from neighbor particles in the film. ${ }^{16}$

Surprisingly, the PC phase containing $1 \mathrm{mM}$ of TTF extracted AuNPs completely from the aqueous phase after vigorous shaking (Fig. 1A). Remarkably, right after shaking the PC-TTF suspension turned red instead of bluish grey (Fig. 1A, middle), as mentioned above. After complete separation of the phases, a dark red-colored solution of AuNPs in PC was obtained (Fig. 1B, red curve). At the same time, the aqueous phase turned yellowish due to the presence of small quantities of the TTF/TTF ${ }^{+\bullet}$ species in PC-saturated water (Fig. 1B, magenta curve). ${ }^{7}$

The PC solution of TTF@AuNPs had both the SPR-component at $532 \mathrm{~nm}$ (red bar) and a small contribution of the SPC-band at $813 \mathrm{~nm}$. Peaks below $480 \mathrm{~nm}$ were assigned to TTF molecules (red vs. blue curve in Fig. 1B). ${ }^{7}$ A tiny SPC-peak observed with TTF@AuNPs at a wavelength above $800 \mathrm{~nm}$ was, most likely, due to the $\pi-\pi$ interaction between neighbouring AuNPs through TTFstacks. In fact, a comparison of SPC-peaks with and without TTF molecules revealed the blue-shift of the SPC-peak (from 813 to $717 \mathrm{~nm}$ ), because the gap between particles in the nanofilm configuration was smaller than that for TTF@AuNPs in PC. Such interactions were enhanced by a concentration effect as AuNPs from $3 \mathrm{~mL}$ of the aqueous phase were transferred into $1 \mathrm{~mL}$ of the oil. Thus, a small part of TTF@AuNPs was aggregated in the PC phase. Furthermore, TTF@AuNPs in PC was concentrated by several subsequent centrifugations to obtain a very dense, black solution. The specific density was estimated to be $1.32 \mathrm{~g} \mathrm{~cm}^{-3}$, which corresponds to $\sim 10 \mathrm{w} \%$ of AuNP loading.

High resolution TEM images were obtained after drying the corresponding solutions, as-prepared citr@AuNPs in water and TTF@AuNPs in PC, on TEM grids (Fig. 1C and D). As the oil phase had an excess of TTF and due to slow evaporation of PC,
$\mathrm{TTF} / \mathrm{TTF}^{+}$formed a thick shell with a distinguishable contrast on the gold surface, which was confirmed by chemical mapping (Fig. 1D). The latter is different from the typical rod-like morphology of TTF upon reduction of gold salts..$^{15,21,22}$

Particle size and $\zeta$-potential distributions are presented in Fig. 1E and F. The initial citr@AuNPs solution had a narrow size distribution with a mean diameter of $36 \mathrm{~nm}$, as determined by DLS. The same particles demonstrated two populations after the transfer into the PC phase and substitution of citrate with TTF: single NPs and their aggregates with mean diameters of 27 and $278 \mathrm{~nm}$, respectively (Fig. 1E).

There are many parameters that could affect the particle size distribution measured by DLS after transfer of the AuNPs into the oil phase. Most likely, a slight deviation of the mean diameter (from 32 to $27 \mathrm{~nm}$ ) was a result of a variation in the physical properties, such as the refractive index, density and viscosity, of the solvent used in comparison with the pure solvent. For example, the reported solubility of water in PC is 8.3-8.6 wt $\% .^{20,23}$ However, according to TEM data (red and orange curves, Fig. 1E) the mean diameters as well as particle size distributions in water and PC are identical (Fig. 1E).

Moreover, both solutions demonstrated a similar mean $\zeta$-potential around $-40 \mathrm{mV}$, which is a bit smaller for the oil phase $(-38 \mathrm{mV})$. In the PC phase, the $\zeta$-potential distribution had a much narrower perfectly bell-shaped distribution in contrast to the very broad distribution in water with several particle populations (Fig. 1F).

As mentioned above, the interaction of AuNPs with electrondonor molecules, such as TTF, led to charging of the gold core and formation of positively charged oxidized electron-donor species that may attach to the surface. ${ }^{24}$ The AuNP core underwent a change of the charge sign during a redox reaction with TTF, as shown in ref. 7 and 25. The initial Fermi level of a single AuNP was below that of bulk gold, so an AuNP was positively 
(A)

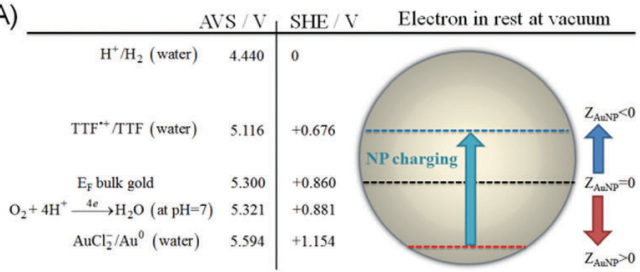

(B)

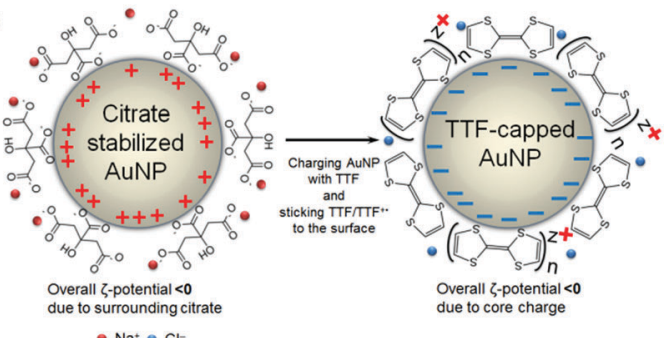

Scheme 1 Schematic representation of the charge distribution on a citr@AuNP and a TTF@AuNP. (A) Explanation of the positive charge for a citr@AuNP and the negative charge for a TTF@AuNP based on the Fermi level equilibration theory. (B) Schematic representation of the recharging process by TTF that leads to retention of the negative $\zeta$-potential.

charged (Scheme 1A). Accumulating electrons from TTF and releasing some TTF/ $\mathrm{TTF}^{+} \bullet$ into the aqueous phase resulted in a negatively charged AuNP core surrounded by $\mathrm{TTF}^{+} \bullet$ and neutral TTF. Also, the positive charge of $\mathrm{TTF}^{+}{ }^{\bullet}$ could be reduced by the presence of $\mathrm{Cl}^{-}$ions in the aqueous phase, therefore, the overall $\zeta$-potential remained negative. Coexistence of $\mathrm{TTF}^{+} \bullet$ and neutral TTF on the surface of AuNPs compensated the repulsive forces between $\mathrm{TTF}^{+} \bullet$ species (Scheme 1B).

Remarkably, the solution of TTF@AuNPs in the PC phase demonstrated long-term stability (weeks) without visible degradation. The interplay between attractive $\pi-\pi$ interactions and Coulombic repulsion among charged particles provides the colloid with exceptional stability, which may be applied to concentrate nanoparticles, as shown above, and to use them in applications such as ink-jet printing.

To sum up, we have observed (i) the self-assembly of AuNPs at the water-PC interface with low interfacial tension without any aggregation promoter or electron-donor molecules such as TTF; and (ii) the extraction of AuNPs from water to the oil phase in the presence of TTF. These observations in comparison with the previously obtained results are summarized in Scheme 2 .

These results raise two questions: (i) what is the role of interfacial tension in the self-assembly of nanoparticles at the liquid-liquid interface? and (ii) to what extent do changes in the three phase contact angle push the nanoparticles to be transferred across the interface?

Theoretical calculations based on thermodynamic energy balance helped in visualizing these effects and highlighted, primarily, the critical role of the interfacial tension in the selfassembly at LLIs.

Unfortunately, the exact solution for the sorption/desorption process of NPs at a LLI is complicated and can be obtained only numerically. ${ }^{17}$ However, Flatte et al. suggested a simplified model to describe interactions between a single nanoparticle

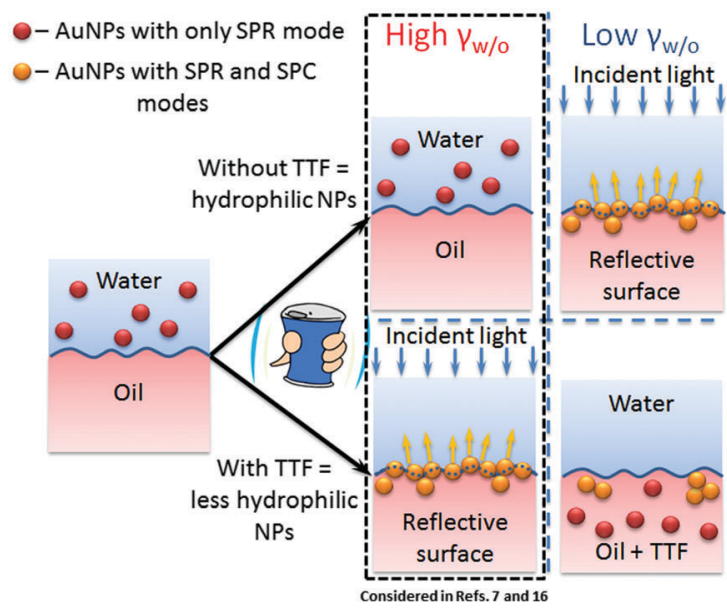

Scheme 2 Similarities and differences between self-assemblies at LLIs with high and low interfacial tensions $\left(\gamma_{w / 0}\right)$. In the case of high $\gamma_{w / o}$ addition of TTF is crucial to obtain a lustrous nanofilm, whereas for a LLI with low $\gamma_{w / o}$ the gold nanoparticles self-assemble into a similar reflective surface without TTF molecules. Addition of TTF promotes transfer of AuNPs into the oil phase and formation of a stable colloid in the oil phase.

and a liquid-liquid interface. ${ }^{17}$ Earlier, Reincke et al. had applied DLVO theory (previously developed by Adamczyk and Weroński in the case of a particle deposition problem) ${ }^{26}$ to understand the self-assembly of charged nanoparticles at a wateroil interface (particularly, water-heptane). ${ }^{27}$ This work took into account several interactions: Coulombic screened (for the aqueous phase) and unscreened (for the oil phase), dipole-dipole (induced dipole in NP due to charge redistribution), as well as van der Waals potentials. However, that description, in comparison to Flatte's one, contains many assumption and not so well-known parameters, but leads to qualitatively similar results. Thus, for the simplicity of understanding, we chose Flatte's model to compare the thermodynamics of adsorption and transfer of AuNPs at the water-PC (low $\gamma_{w / 0}$ ) and water-DCE (high $\gamma_{w / 0}$ ) interfaces in the presence and absence of TTF (Section S4 in the ESI $\dagger$ ).

In the model, Flatte et al. ${ }^{17}$ considered energies devoted only to capillary forces, changing of the solvation sphere from the aqueous to the oil phase and the line tension, which contains all kinds of interactions pushing NPs away from the interface. Thus, the three-phase contact angle $(\theta)$ and the charge of the NP $(Z)$ were tunable parameters. A detailed description and calculation results are given in Section S4 of the ESI, $\dagger$ while here we highlight only the main findings.

Calculations revealed a significantly deep well located at the interface for $32 \mathrm{~nm}$ AuNPs for bare water-organic interfaces, with both high (w-DCE) and low (w-PC) interfacial tensions. For DCE (Fig. S4A and B in the ESI $\dagger$ ), the well was separated from the bulk by a relatively large $\left(c a .1000 k_{\mathrm{B}} T\right)$ potential barrier. It makes the landing of hydrophilic NPs at the interface impossible and, thus, no film is formed, which corroborates our experimental results. Nevertheless, for the PC-water interface, the potential barrier was less than $100 k_{\mathrm{B}} T$. It can be overcome during emulsification, when formation of additional surfaces lowers the barrier height and makes attachment of the particles to the LLI more favorable. 
Reduction of AuNPs by TTF significantly altered the energetic profiles, such as the height of the potential barrier and the depth of the well (Fig. S4C and D in the ESI $\dagger$ ). Also, the presence of TTF on the AuNP surface makes them less hydrophilic. For the water-DCE interface, it led to a deeper potential well and stabilization of the whole system in the nanofilm state. In the case of PC-water, the potential barrier on the aqueous side disappeared and AuNPs could be easily extracted into the oil phase. Of course, the PC phase $\left(\varepsilon_{\mathrm{PC}}=64\right)$ has a higher dielectric constant than DCE $\left(\varepsilon_{\mathrm{DCE}}=10\right)$, which significantly facilitates transfer of partially charged AuNPs to the oil phase or their assembly at the interface.

We demonstrated that the interfacial tension has a crucial role in the self-assembly of charged particles due to its contribution to these potential barriers, whereas wettability (three-phase contact angle) is still important as a secondary parameter that may help in adjusting the position of nanoparticles at the interface. The obtained results are also applicable to explain spontaneous self-assembly of AuNPs for other LLIs, such as water-dimethyl carbonate, ${ }^{28,29}$ or in the case of alcohol-assisted self-assembly where alcohols are used to decrease the interfacial tension. ${ }^{30}$

To sum up, the interface between water and PC is an interesting alternative to perform self-assembly at a liquid-liquid interface. The extremely low interfacial tension of $c a .3 \mathrm{mN} \mathrm{m}^{-1}$ allows selfassembly of AuNPs without any "promoter" in both phases. In the presence of TTF, the AuNPs were transferred easily into the oil phase with the formation of a stable dense colloid. Such colloidal solutions may be of interest to obtain dense gold-inks or standard samples for electron microscopy. Qualitative calculations explained quite well the experimental observations of interfacial self-assembly and put them in line with previously published results.

\section{Notes and references}

1 M. P. Cecchini, V. A. Turek, J. Paget, A. A. Kornyshev and J. B. Edel, Nat. Mater., 2012, 12, 165-171.

2 J. B. Edel, A. A. Kornyshev and M. Urbakh, ACS Nano, 2013, 7, 9526-9532.

3 H. Duan, D. Wang, D. G. Kurth and H. Mohwald, Angew. Chem., Int. Ed., 2004, 116, 5757-5760.

4 W. H. Binder, Angew. Chem., Int. Ed., 2005, 44, 5172-5175.
5 V. A. Turek, M. P. Cecchini, J. Paget, A. R. Kucernak, A. A. Kornyshev and J. B. Edel, ACS Nano, 2012, 6, 7789-7799.

6 M. P. Konrad, A. P. Doherty and S. E. J. Bell, Anal. Chem., 2013, 85, 6783-6789.

7 E. Smirnov, M. D. Scanlon, D. Momotenko, H. Vrubel, M. A. Méndez, P.-F. Brevet and H. H. Girault, ACS Nano, 2014, 8, 9471-9481.

8 K. Y. Lee, G.-W. Cheong and S. W. Han, Colloids Surf., A, 2006, 275, 79-82.

9 M. Tebbe, S. Lentz, L. Guerrini, A. Fery, R. A. Alvarez-Puebla and N. Pazos-Perez, Nanoscale, 2016.

10 Y.-K. Park and S. Park, Chem. Mater., 2008, 20, 2388-2393.

11 S. G. Booth and R. A. W. Dryfe, J. Phys. Chem. C, 2015, 119, 23295-23309.

12 J. B. Edel, A. A. Kornyshev, A. R. Kucernak and M. Urbakh, Chem. Soc. Rev., 2016, 45, 1581-1596.

13 M. D. Scanlon, P. Peljo, M. A. Méndez, E. Smirnov and H. H. Girault, Chem. Sci., 2015, 6, 2705-2720.

14 A. R. Siedle, G. A. Candela, T. F. Finnegan, R. P. Van Duyne, T. Cape, G. F. Kokoszka, P. M. Woyciejes and J. A. Hashmall, Inorg. Chem., 1981, 20, 2635-2640.

15 K. Naka, D. Ando, X. Wang and Y. Chujo, Langmuir, 2007, 23, 3450-3454.

16 E. Smirnov, P. Peljo, M. D. Scanlon, F. Gumy and H. H. Girault, Nanoscale, 2016, 8, 7723-7737.

17 M. E. Flatté, A. A. Kornyshev and M. Urbakh, J. Phys.: Condens. Matter, 2008, 20, 73102.

18 A. G. Williamson and N. F. Catherall, J. Chem. Eng. Data, 1971, 16, 335-336.

19 J. A. Riddick, W. B. Bunger and T. K. Sakano, Techniques of Chemistry 4th ed., Volume II. Organic Solvents, John Wiley and Sons, New York, NY, 4th edn, 1985.

20 Huntsman Corporation, 2011.

21 J. Puigmartí-Luis, D. Schaffhauser, B. R. Burg and P. S. Dittrich, Adv. Mater., 2010, 22, 2255-2259.

22 Y. Xing, A. Wyss, N. Esser and P. S. Dittrich, Analyst, 2015.

$23 \mathrm{http} / / / \mathrm{macro} .1$ su.edu/HowTo/solvents/Propylene Carbonate.htm.

24 E. Smirnov, P. Peljo, M. D. Scanlon and H. H. Girault, ACS Nano, 2015, 9, 6565-6575.

25 E. Smirnov, P. Peljo, M. D. Scanlon and H. H. Girault, Electrochim. Acta, 2016, 197, 362-373.

26 Z. Adamczyk and P. Weroński, Adv. Colloid Interface Sci., 1999, 83, 137-226.

27 F. Reincke, W. K. Kegel, H. Zhang, M. Nolte, D. Wang, D. Vanmaekelbergh and H. Mohwald, Phys. Chem. Chem. Phys., 2006, 8, 3828-3835.

28 K. Zhang, J. Ji, Y. Li and B. Liu, Anal. Chem., 2014, 86, 6660-6665.

29 K. Zhang, J. Zhao, H. Xu, Y. Li, J. Ji and B. Liu, ACS Appl. Mater. Interfaces, 2015, 7, 16767-16774.

30 F. Reincke, S. G. Hickey, W. K. Kegel and D. Vanmaekelbergh, Angew. Chem., Int. Ed., 2004, 43, 458-462. 